\title{
SITES: Solar Iterative Temperature Emission Solver for Differential Emission Measure Inversion of EUV Observations
}

\author{
Huw Morgan ${ }^{1}$ (D) James Pickering ${ }^{1}$
}

Received: 15 May 2019 / Accepted: 8 September 2019 / Published online: 1 October 2019

(C) The Author(s) 2019

\begin{abstract}
Extreme ultraviolet (EUV) images of the optically-thin solar corona in multiple spectral channels give information on the emission as a function of temperature through differential emission measure (DEM) inversions. The aim of this paper is to describe, test, and apply a new DEM method named the Solar Iterative Temperature Emission Solver (SITES). The method creates an initial DEM estimate through a direct redistribution of observed intensities across temperatures according to the temperature response function of the measurement, and iteratively improves on this estimate through calculation of intensity residuals. It is simple in concept and implementation, is non-subjective in the sense that no prior constraints are placed on the solutions other than positivity and smoothness, and can process a thousand DEMs a second on a standard desktop computer. The resulting DEMs replicate model DEMs well in tests on Atmospheric Imaging Assembly/Solar Dynamics Observatory (AIA/SDO) synthetic data. The same tests show that SITES performs less well on very narrow DEM peaks, and should not be used for temperature diagnostics below $\approx 0.5 \mathrm{MK}$ in the case of AIA observations. The SITES accuracy of inversion compares well with two other established methods. A simple yet powerful new method to visualize DEM maps is introduced, based on a fractional emission measure (FEM). Applied to a set of AIA full-disk images, the SITES method and FEM visualization show very effectively the dominance of certain temperature regimes in different large-scale coronal structures. The method can easily be adapted for any multi-channel observations of optically-thin plasma and, given its simplicity and efficiency, will facilitate the processing of large existing and future datasets.
\end{abstract}

Keywords Image processing · Corona

\section{Introduction}

Understanding the physics of the Sun's atmosphere demands increasingly detailed and accurate observations. The development of new analysis methods to gain physical observables

\author{
H. Morgan \\ hmorgan@aber.ac.uk \\ 1 Physics Department, Aberystwyth University, Ceredigion, SY23 3BZ, Cymru, UK
}


from remote sensing observations is an ongoing and critically important effort. As part of this effort, this paper presents a new Differential Emission Measure (DEM) method for the temperature/density analysis of solar coronal optically-thin emission lines. The extreme ultraviolet (EUV) spectrum from the solar atmosphere contains several strong emission lines from highly ionized species above a relatively low background. These lines are emitted from the hot corona only, thus narrowband EUV observations are an excellent probe of the low corona, with little contamination from the underlying photosphere and lower atmosphere.

The concept of using EUV line intensities to estimate the temperature of the emitting plasma is based on the temperature of formation of the line: a range of temperatures at which a certain ion can exist, and the relative population of that ion as a function of temperature. Thus calibrated observations of two lines with different formation temperatures can give a constraint on the dominant plasma temperature. Based on this concept, the simplest approach to estimating a dominant coronal temperature is the line ratio method, which assumes an isothermal plasma (see, for example, the description and criticism of Weber et al., 2005).

In the general case, imaging instruments provide an observed intensity integrated across a narrow bandpass that spans one or more spectral lines - this is the case for an EUV imaging instrument such as the Atmospheric Imaging Assembly onboard the Solar Dynamics Observatory (AIA/SDO). Thus the temperature response of each channel may be computed based on the wavelength response of that channel and modeled line intensities from an established atomic database (such as CHIANTI, Dere et al., 1997) using certain assumptions (e.g. Maxwell-Boltzmann distributions and thermal equilibrium). The measured intensity of multiple bandpasses, or channels, with different temperature responses, allow the estimation of emission as a function of temperature, or a DEM. A DEM is a powerful characterization of the coronal plasma - it is an estimate of the total number of electrons squared along the observed line of sight (similar to a column mass) at a given temperature. The DEM method has revealed the general temperature characteristics of the main structures seen in the corona; for example, closed-field active regions are hot and multithermal ( $>2 \mathrm{MK}$ ), open-field regions are colder $(<1.1 \mathrm{MK})$, and in between is the quiet corona $(\approx 1.4 \mathrm{MK})$ (Del Zanna, 2013; Hahn, Landi, and Savin, 2011; Mackovjak, Dzifčáková, and Dudík, 2014; Hahn and Savin, 2014). Changes in DEM over time are related to heating or cooling, and can be applied over large datasets to reveal solar cycle trends (Morgan and Taroyan, 2017).

For an imaging instrument such as AIA, the DEM method inverts measured intensities in a small number of bandpasses to give the emission as a function of temperature across a large number of temperature bins. This is an underdetermined problem that requires additional constraints on the solution, such as positivity and smoothness. There are several types of DEM methods in use, well summarized in the introduction to Hannah and Kontar (2012). One method is that of Hannah and Kontar (2012), which uses Tikhonov regularization to find an optimal weighting between fitting the data and satisfying additional constraints of positivity of the DEM (negative emission is unphysical), minimizing the integrated emission and smoothness of the result. To our knowledge, the most computationally fast method is that of Cheung et al. (2015), based on simplex optimization of a set of smooth basis functions, or a sparse matrix. Plowman, Kankelborg, and Martens (2013) use a parametric functional form for the DEM, solved with a regularized inversion combined with an iterative scheme for removal of negative DEM values. A similar parametric form is also used by Nuevo et al. (2015) in the context of coronal tomography and a localized DEM.

This work presents a new DEM inversion method in Section 2. The method is introduced in the context of the type of imaging observations made by an instrument such as AIA, but can easily be generalized to any observation where the measurement temperature response 
Figure 1 (a) The temperature response of the seven AIA EUV channels, as given by the standard AIA calibration routines in SolarSoftware, based on CHIANTI atomic data and normalized through cross-calibration with EVE data. This set is for date 01 January 2011. (b) The relative response as a function of temperature. At a given temperature, the relative responses sum to unity over all channels.

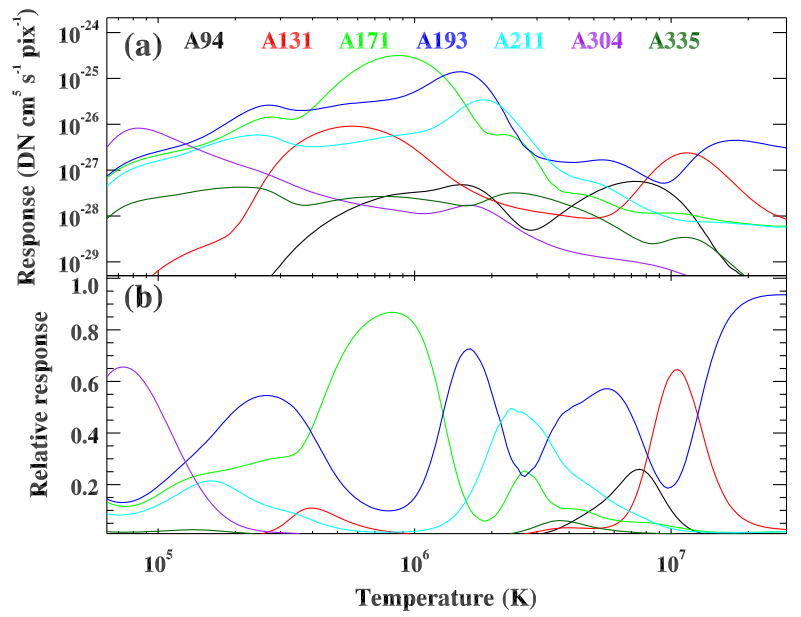

is known. Tests of the method on synthetic observations made from model DEMs are made in Section 3, along with a non-rigorous test on computation time. Section 4 discusses uncertainty in AIA measurements, and applies the method to data. An effective method to visualize DEM maps is also presented in Section 4. A brief summary is given in Section 5.

\section{The DEM Method}

A set of intensities $I_{0}, I_{1}, \ldots, I_{n-1}$ are measured by $i=0,1, \ldots, n-1$ AIA channels, with associated errors $\sigma_{i}$. The response of each channel as a function of temperature, $R_{i j}$, is known for a set of temperature bins indexed $j=0,1, \ldots, n_{t}-1$. This work uses the response functions as given by the standard AIA SolarSoftware routines, calculated from the CHIANTI atomic database (Dere et al., 1997; Landi et al., 2012), cross-calibrated over time with the Extreme Ultraviolet Variability Experiment (EVE/SDO) observations and including a correction to the $94 \AA$ channel calibration (Boerner et al., 2014). An example of these functions are shown in Figure 1a. We assume that each response function has a relative error, $\varepsilon_{i}$, constant over all temperatures. These uncertainties are discussed in a following section.

Before considering weightings associated with the relative noise in each channel, we first introduce the simple concept of relative temperature responses. The relative response for a given channel (indexed $i$ ) and temperature (indexed $j$ ), $S_{i j}$, is calculated as

$$
S_{i j}=\frac{R_{i j}}{\sum_{i=0}^{n-1} R_{i j}},
$$

so that, at a given temperature bin, the relative responses sum to unity over all channels. The relative responses are an useful value, and are shown in Figure 1b. For example, at very high temperatures (>10 MK), the relative response of the $193 \AA$ A channel is almost 1 , showing that any DEM method using solely AIA data will be very uncertain at these flare temperatures, since only one channel is giving information at this temperature. A similar argument holds for low $(<0.1 \mathrm{MK})$ temperatures, where the relative response of the $304 \AA$ channel increases to 0.7 . The relative response (further weighted by the relative error in each channel, to be introduced later) is used in the DEM method to combine the information 
from each channel. Thus, as can be seen from Figure 1b, at temperatures near 0.9 MK, the resulting DEM will be dominated by the $171 \AA$ A channel.

It is convenient to include information on the relative measurement error of each channel and the estimated errors of the response functions in the relative response. Thus Equation 1 becomes

$$
S_{i j}=\frac{w_{i} R_{i j}}{\sum_{i=0}^{n-1} w_{i} R_{i j}},
$$

where $w$ is a weighting based on the measurement and calibration errors,

$$
w_{i}=\frac{1}{\sqrt{\left(\frac{\sigma_{i}}{I_{i}}\right)^{2}+\varepsilon_{i}^{2}}} .
$$

Thus channels with smaller relative errors (i.e. higher signal to noise and/or less uncertainty in response function) will have greater weighting in the DEM estimate.

An initial DEM estimate, $D_{j}$, is given by

$$
D_{j}=\left[\sum_{i}^{n-1} S_{i j}\left(I_{i} \frac{R_{i j} \Delta T_{j}}{\sum_{j}^{n_{t}-1}\left(R_{i j} \Delta T_{j}\right)^{2}}\right)\right] \otimes K,
$$

where $K$ is a smoothing kernel and the $\otimes$ is the convolution operator. In words, a set of $n$ DEM profiles is calculated, one for each channel, based directly on the response function of each channel (the expression within the round brackets). Since the observed intensity is distributed over the DEM temperature range according to the response function of that channel, integrating these individual DEMs over temperature would result in exactly the observed intensities. These individual DEMs are combined into a single DEM through a weighted mean, using the weighting of the relative responses (i.e. product with the relative responses, $S_{i j}$, and summation over $i$ ). This DEM is convolved with a narrow Gaussian kernel over temperature, $K$, to ensure a smooth DEM. The kernel $K$ is a Gaussian profile in logarithmic temperature, with a width (standard deviation) of 3.2 bins in logarithmic temperature, for 43 temperature bins over a temperature range of $0.07-20 \mathrm{MK}$. These values are found through trial and error with the criteria that the smoothing width is kept at a minimum value whilst still resulting in smooth DEMs. The width of the smoothing kernel is, in fact, the only subjective choice in this procedure. If more temperature bins are set, then the width of the kernel should be increased in proportion.

From this initial DEM, a set of modeled intensities $M_{i}$ is computed for each channel by

$$
M_{i}=\sum_{j}^{n_{t}-1} D_{j} R_{i j} \Delta T_{j}
$$

The residual, or difference between the observed and modeled intensities, is calculated as $I_{i}^{\prime}=I_{i}-M_{i}$. This residual intensity is fed back into Equation 4 (taking the place of $I_{i}$ in the equation), and the resulting residual DEM added to the previous DEM. At this step, the main DEM is thresholded to a minimum value of zero since the residuals may result in a negative DEM at certain temperatures - thus a positivity constraint is applied. This process is iterated until convergence is reached, defined as when the weighted mean of the absolute ratios between the measurement residuals at the current iteration and the initial measurement, drops below an appropriately small threshold, for example $1 \%$. The weights for this mean 
are those given by Equation 3. This is a sensible criterion for convergence - the process stops when the mean changes to the output DEM become small, with weighting towards the higher certainty measurements. There are similarities in this iterative approach to that of Plowman, Kankelborg, and Martens (2013), which computes residual data intensities at several iterations in order to adjust the estimated DEM and eliminate negative intensities. However, the core DEM estimation at each iteration, given by Equation 4, is quite different to their method.

An estimate for the DEM error, $d$, at each temperature bin $j$ is

$$
d_{j}=\sqrt{\sum_{i}^{n-1} S_{i j}\left[\left(\sigma_{i} / I_{i}\right)^{2}+\varepsilon_{i}^{2}\right]} .
$$

The relative measurement error, $\sigma_{i} / I_{i}$, and the response function relative uncertainty, $\varepsilon_{i}$, are summed in quadrature, giving the total squared measurement error for each channel. These are multiplied by the relative response $S_{i j}$ in order to distribute over temperatures, and summed over all channels, corresponding to the equivalent steps in the DEM estimate of Equation 4. The square root of this value gives the final DEM uncertainty. A complete error propagation treatment should consider the smoothing kernel and multiple iterations, but these steps would defeat the aim of implementing an efficient method. The uncertainties given by the simple calculation of Equation 6 give values that agree well with tests involving varying the input measurements according to measurement noise, as is shown in Section 3.4.

\section{Demonstration Using Synthetic Data}

\subsection{A Simple Test}

A model DEM is produced by the Gaussian

$$
D^{\prime}=A \exp \left(\left[\frac{t-t_{c}}{w_{t}}\right]^{2}\right),
$$

with peak maximum $A=1.4 \times 10^{21} \mathrm{~cm}^{-5} \mathrm{~K}^{-1}$, central temperature $t_{c}=1.4 \mathrm{MK}$, and width $w_{t}=0.9 \mathrm{MK}$. Using the AIA response functions (as shown in Figure 1), synthetic observations are created for the seven channels, in units of $\mathrm{DN} \mathrm{cm} \mathrm{s} \mathrm{s}^{-1} \mathrm{pix}^{-1}$ by integrating the product of the DEM with the response functions over temperature. Measurement uncertainties are given by the AIA SolarSoftware routine aia_bp_estimate_error. The synthetic observations are input into SITES, using 43 temperature bins within a temperature range of $0.07-20 \mathrm{MK}$, with a regular bin size in logarithmic temperature. The method terminates at 101 iterations when convergence, as defined in the method, reaches $1 \%$. To avoid edge effects caused by the smoothing truncation, the first and last DEM bins are discarded, leaving 41 temperature bins in the results. The maximum absolute relative difference between input target intensities and the method derived intensities (the $M_{i}$ of Equation 5) is $3.5 \%$ for the $335 \AA$ channel. The mean absolute measurement difference, $T_{I}$, over all channels is defined as

$$
T_{I}=\frac{1}{n} \sum_{i}^{n-1} \frac{\left|I_{i}-M_{i}\right|}{I_{i}},
$$

and is $1.2 \%$ for this simple test. 
Figure 2 Comparing input (black) and output (red) DEM curves for the simple case of a single Gaussian in temperature (Equation 7). The light red error bars show the uncertainty in the fitted DEM.

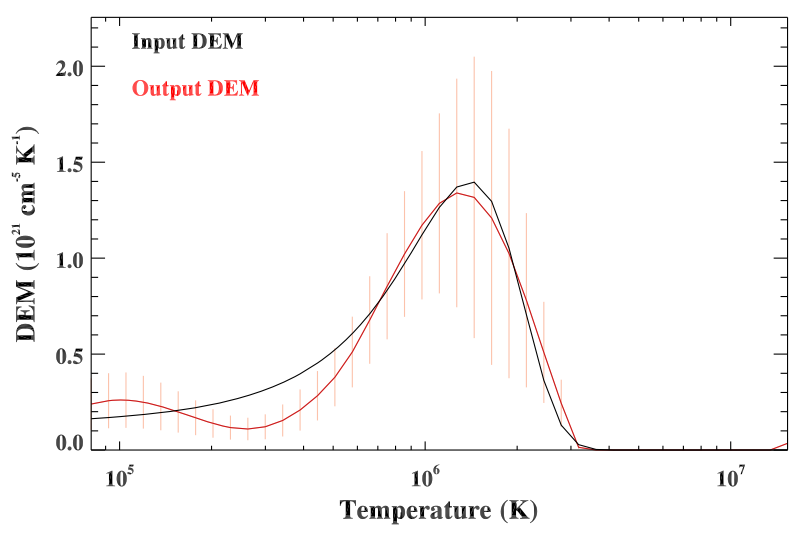

The resulting DEM is compared to the target input DEM in Figure 2. The median absolute relative DEM deviation, $T_{D}$, between the input DEM, $D^{\prime}$, and output estimated DEM, $D$, is defined as

$$
T_{D}=\operatorname{median} \frac{\left|D_{j}^{\prime}-D_{j}\right|}{D_{j}^{\prime}},
$$

and is $26 \%$ for this simple test. The median is calculated over temperature bins $j$ and is used here rather than the mean to avoid the influence of very small, or zero, values of input DEM at some temperatures bins.

The correlation, $c$, between the input and output DEM curves is defined as

$$
c=\frac{\sum_{j}^{n_{t}-1}\left(D_{j}-\bar{D}_{j}\right)\left(D_{j}^{\prime}-\overline{D_{j}^{\prime}}\right)}{\sqrt{\sum_{j}^{n_{t}-1}\left(D_{j}-\bar{D}_{j}\right)^{2} \sum_{j}^{n_{t}-1}\left(\bar{D}_{j}^{\prime}-\overline{D_{j}^{\prime}}\right)^{2}}}
$$

and is $98 \%$ for this simple test. Thus the position and width of the main peak is well fitted.

One concern is the range of temperatures used for the calculation. The examples here have temperatures limited to between 0.07 and $20 \mathrm{MK}$. Some channels have significant values in their response functions outside this range, leading to an inherent uncertainty that can be included in the estimate of each response function uncertainty. Thus an estimate of the relative uncertainty in the response of each channel is given by

$$
\varepsilon_{i}=\sqrt{e_{i}^{2}+\left(\frac{\sum_{j 0} R_{i j} \Delta T_{j}}{\sum_{j 1} R_{i j} \Delta T_{j}}\right)^{2}},
$$

where $e_{i}$ is the calibration uncertainty for each channel, and the subscript $j 1$ are the indices of temperature bins included within the temperature range, and $j 0$ otherwise. The $e_{i}$ are given by AIA SolarSoftware routines, and are $50 \%$ for the 94,131 , and $304 \AA$ channels, $25 \%$ otherwise. $\varepsilon_{i}$ is a large uncertainty, ranging from $27 \%$ for the $171 \AA$ channel to $103 \%$ for the $131 \AA$ channel. Channels with large contributions to their response functions outside of the temperature range of interest have a lesser weighting in calculating the final DEM.

The single-Gaussian DEM is used as a test of SITES across a broad range of Gaussian central temperature and Gaussian widths. The central temperature is increased from $\log T=$ 5.3 to 7.05 in 160 increments and the widths from $\log T=0.1$ to 0.35 in 160 increments (note this differs from the example of Figure 2, which is formed from Gaussians in linear 

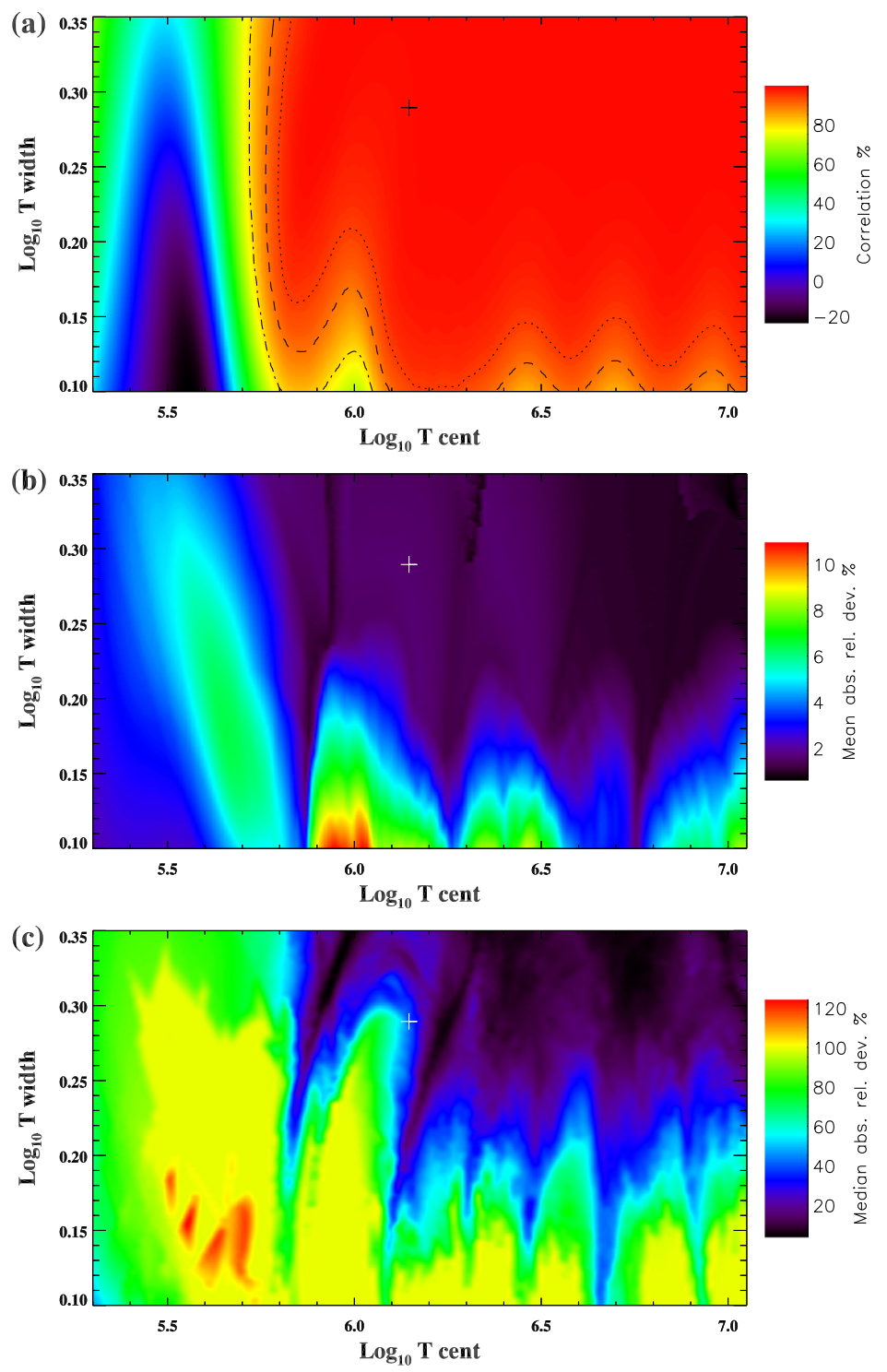

Figure 3 (a) Correlation $c$ between input and SITES-inverted DEM profiles. (b) Mean absolute relative deviation, $T_{I}$, between input measurement and output fitted measurement. (c) Median absolute relative deviation, $T_{D}$, of input and SITES-inverted DEM profiles. These are calculated for a range of centers and widths in logarithmic temperature of single-Gaussian DEM profiles. The dotted, dashed, and dot-dashed lines in (a) show the 95, 90, and $80 \%$ correlation levels, respectively. The cross symbol shows the position corresponding to the single-Gaussian example shown in Figure 2.

temperature). For each input DEM, synthetic measurements are calculated and given as input to SITES, as above for Figure 2. The correlation between input and output DEM, $c$, as given by Equation 10 is calculated, giving a measure of the similarities of the profiles. This is shown in Figure 3a. A broad range of central temperatures and widths bounded by the 
Figure 4 Comparing input (black) and output (red) DEM curves for the complex case of two Gaussians in temperature and a constant background. The light red error bars show the uncertainty in the output DEM.

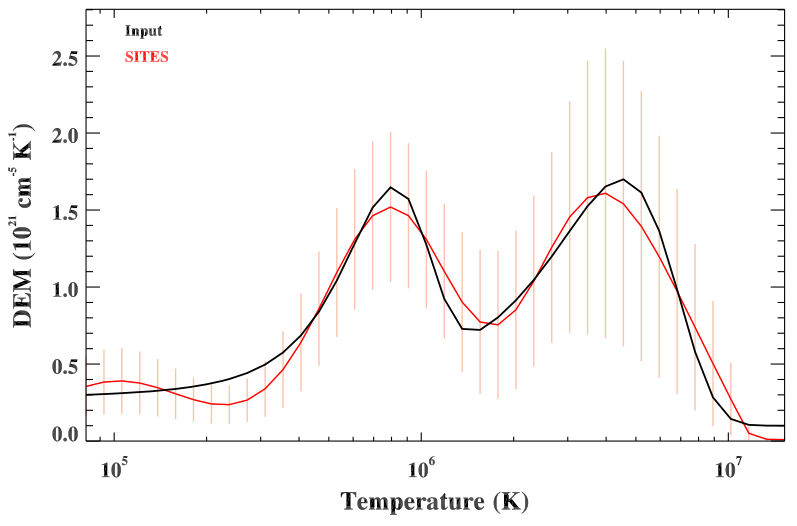

dotted line give correlations above $95 \%$. Poor correlations, below $80 \%$, are found for low temperatures below $\log T=5.7$, and for very narrow profiles at all central temperatures. Figure $3 \mathrm{~b}$ shows the mean absolute relative deviation of the input and output measurements, $T_{I}$, as given by Equation 8 . The worst match, close to $10 \%$ deviation, is found for low temperatures or higher temperatures at narrow widths. The deviation otherwise is good, with the majority of the parameter space at values of $4 \%$ or lower. This is to be expected, given that the iterative scheme is designed to reduce this deviation. Figure $3 \mathrm{c}$ shows the median absolute relative deviation between the input and output DEMs, $T_{D}$, as given by Equation 9 . For the broad region dominated by very high correlations, the deviation is around $15-50 \%$. This deteriorates to over $50 \%$ for low temperatures below $\log T=5.7$, or for narrow profiles at all temperatures.

In summary, SITES performs poorly for narrow DEM profiles at all temperatures. This is inherent to estimating DEMs from an instrument such as AIA, regardless of the method, given the broad multiple-peaked temperature profiles in most channels. SITES performs very poorly for DEMs peaked at cool temperatures below $\log T=5.7(\approx 0.5 \mathrm{MK})$. At higher temperatures, and broader peaks, SITES performs very well, with $95 \%$ correlation with the target input DEMs.

\subsection{A Complex Test}

A more complex model DEM sums two Gaussian peaks over a constant background. The background emission has a value of $10^{20} \mathrm{~cm}^{-5} \mathrm{~K}^{-1}$, and the Gaussian peaks have amplitude $A=[1.6,1.6] \times 10^{21} \mathrm{~cm}^{-5} \mathrm{~K}^{-1}$, centered at temperatures $t_{c}=[0.8,4.5] \mathrm{MK}$, with widths $w_{t}=[0.35,3.0]$ MK. Synthetic observations are created from this model DEM as for the simple case above. The comparison between input and output DEM is shown in Figure 4. The number of iterations is 114 and $T_{I}$ is $1.3 \%$, with a maximum deviation of $3.4 \%$ for the $94 \AA$ channel. $T_{D}$ is $12 \%$. The position of the two peaks in the resulting DEM estimate agree well with the input DEM - the method is effective at finding these peaks, reflected in the $c=97.7 \%$ correlation between input and output DEM.

The performance of SITES is tested for various combinations of parameters for the twoGaussian plus background DEM profile through varying the central temperature of each peak. This experiment is repeated for two cases of wide and narrow Gaussians. The Gaussians are formed in logarithmic temperature (note this differs from the example of Figure 4, which is formed from Gaussians in linear temperature). Figure 5 shows four characteristic examples of the two-Gaussian parameter space. Figure $5 \mathrm{a}$ is for a cool peak at $\log T=5.5$ 
Figure 5 DEM profiles formed from two Gaussians in logarithmic temperature plus a constant background. The black lines are the input DEM and the red lines are the SITES DEM.

The solid (dashed) lines are for wide (narrow) Gaussian profiles ( 0.35 and 0.1 in logarithmic temperature, respectively). Four examples are shown here for the logarithmic peak temperatures of (a) 5.5 and 6.55 , (b) 5.5 and 7.0, (c) 6.2 and 6.55, and (d) 6.2 and 7.0. The vertical dashed lines show the central temperature of each peak.
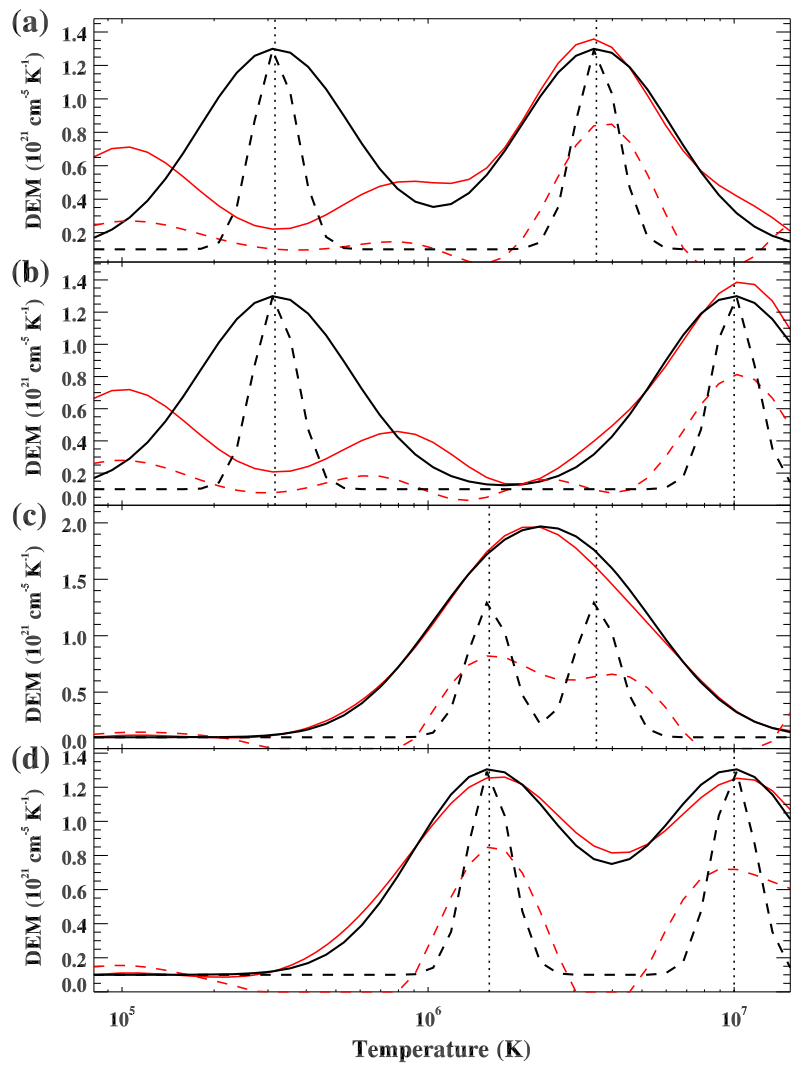

and a hot peak at $\log T=6.55$. For the wide Gaussian profiles (solid lines), the hot peak is well-fitted by SITES, but the fit for the cool peak is poor. The same holds for the narrow Gaussians (dashed lines). The position of the hot narrow peak is found by SITES, although the method struggles to fit the profile properly, with regions next to the peak at zero emission. Figure $5 \mathrm{~b}$ is for a cool peak at $\log T=5.5$ and a hot peak at $\log T=7.0$, with similar results to Figure 5a. Figure $5 \mathrm{c}$ is for a cool peak at $\log T=6.2$ and a hot peak at $\log T=6.55$, thus the wide Gaussians are blended. SITES fits this profile very well. There are two closelyplaced yet distinct peaks in the narrow Gaussian DEM. The SITES DEM also shows two peaks, but is far smoother than the target DEM. Note also the tendency for regions close to the two peaks to have zero emission. Figure $5 \mathrm{~d}$ is for a cool peak at $\log T=6.2$ and a hot peak at $\log T=7.0$. Similar to the previous case, the wide DEMs are fitted very well. The narrow peaks are found by SITES, but are smoother, and tend to zero in nearby regions.

Figure 6 shows the performance of SITES for a range of central temperatures for both Gaussian peaks, for the case of the wide Gaussians. The correlation between the input and SITES DEMs, shown in Figure 6a, is excellent $(c>80 \%)$ for all peak hot temperatures, and cool temperatures above $0.5 \mathrm{MK}$. Below this cool temperature, the performance of SITES is poor, despite the close fit to the input measurement as shown in Figure $6 \mathrm{~b}$. The same poor fit for low temperatures is seen in the median absolute relative deviation of the input and output DEMs in Figure 6c.

Figure 7 shows the same parameter test for narrow Gaussian profiles. Overall, the correlation, fit to measurement, and fit to the DEM have deteriorated throughout the parameter 
(a)
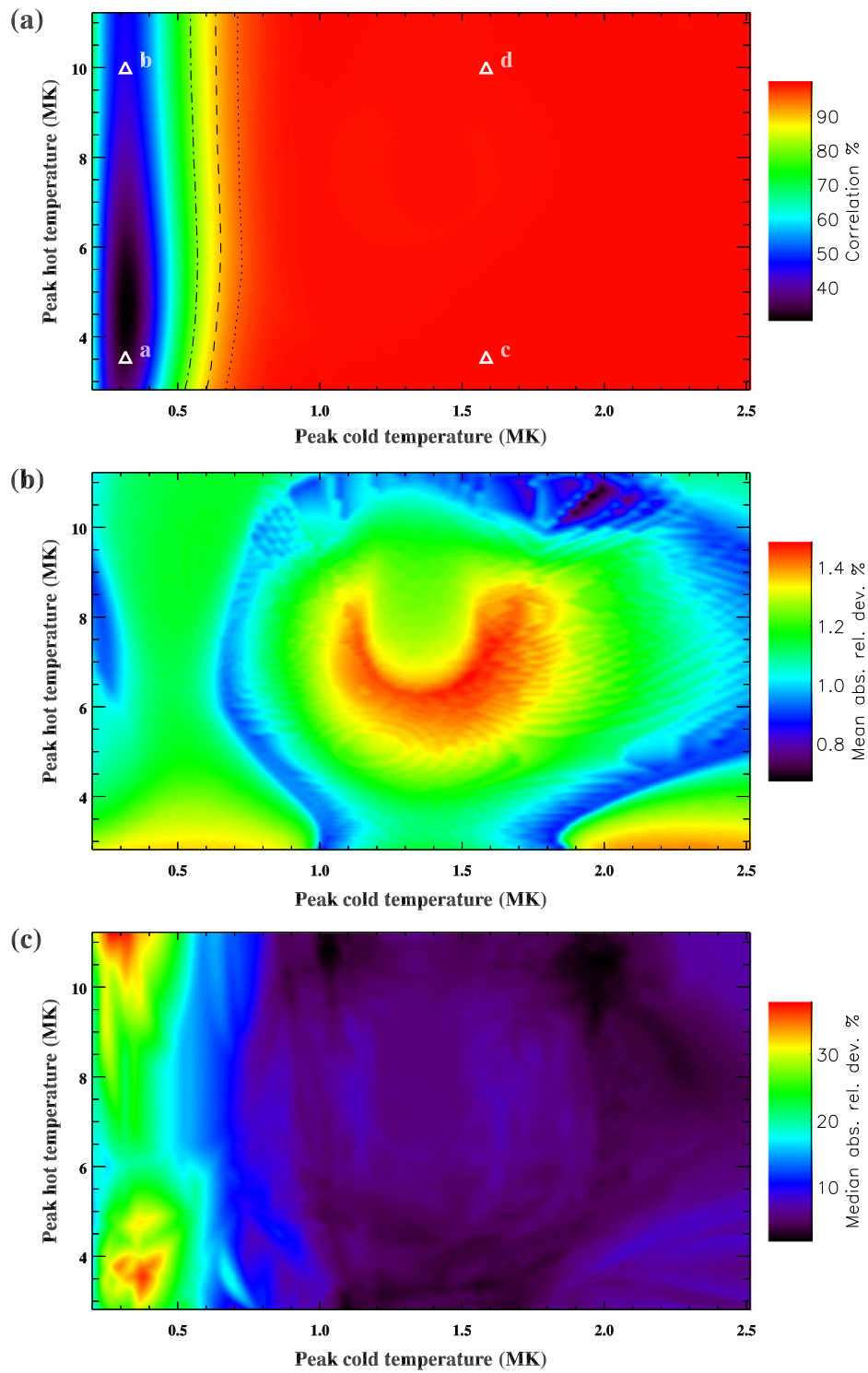

Figure 6 (a) Correlation $c$ between input and SITES-inverted DEM profiles. (b) Mean absolute relative deviation, $T_{I}$, between input measurement and output fitted measurement. (c) Median absolute relative deviation, $T_{D}$, of input and SITES-inverted DEM profiles. These are calculated for a range of central peak temperatures for two wide Gaussian DEM profiles, with the $x$-axis ( $y$-axis) corresponding to the central temperature of the cooler (hotter) peak. The four triangle symbols labeled a-d in (a) correspond to the four example profiles of Figure 5a-d. The dotted, dashed, and dot-dashed lines in (a) show the 95, 90, and 80\% correlation levels, respectively.

space. The very poor fit at low cold peak temperatures remains. In summary, the conclusions for a complex double-Gaussian DEM profile are similar to the case of a single Gaussian in the previous section. SITES performs poorly for narrow DEM profiles at all tempera- 

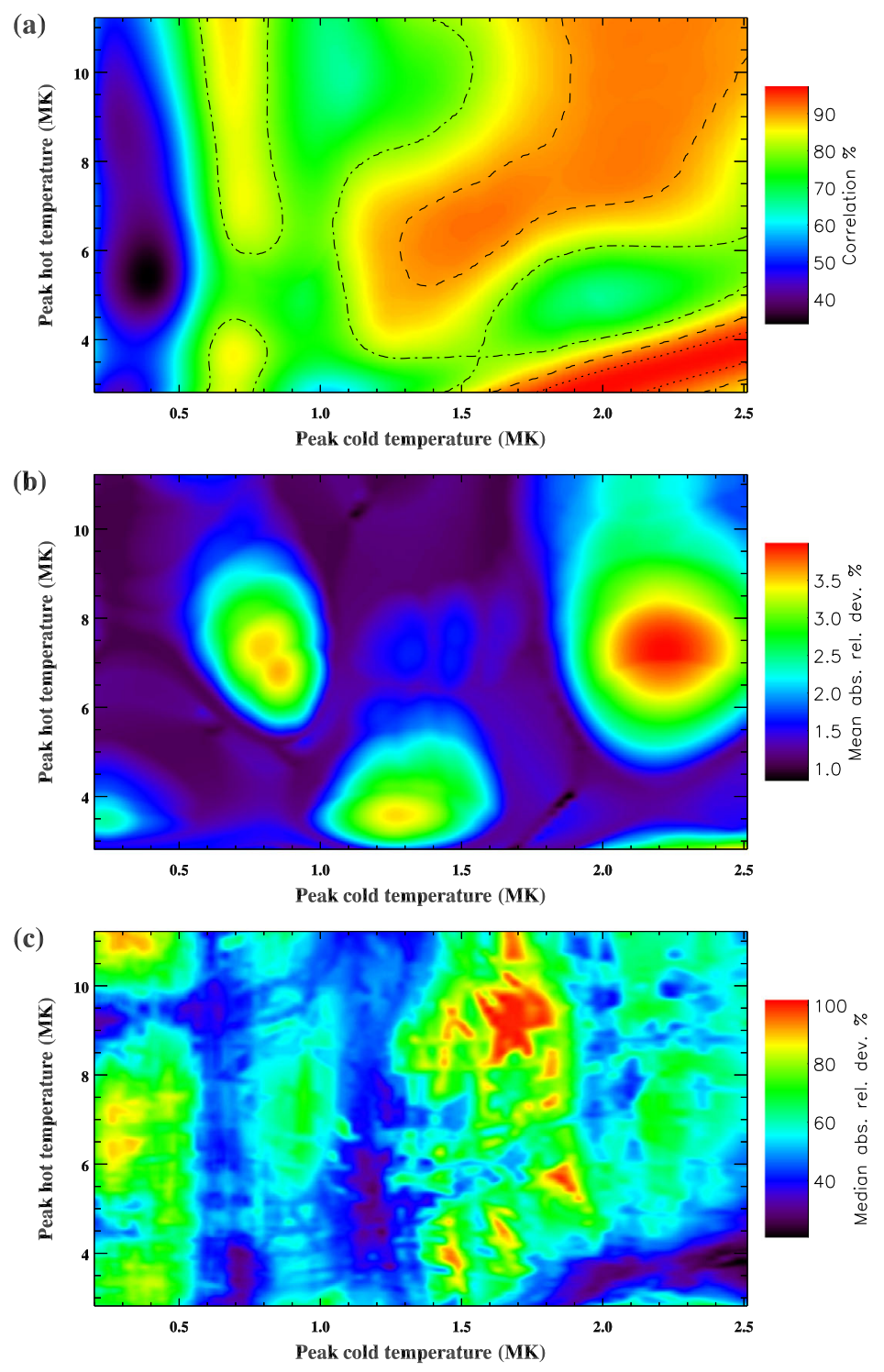

Figure 7 Same as Figure 6, but for the two narrow Gaussians.

tures, and performs very poorly for DEMs which contain peaks at cool temperatures below $\log T=5.7$ ( $\approx 0.5 \mathrm{MK})$. At higher temperatures, and broader peaks, SITES performs very well, with $c=95 \%$ correlation with the target input DEMs.

\subsection{Computational Speed and Convergence Threshold}

To test computational efficiency, SITES is applied 1000 times to the complex 3-Gaussian plus background DEM distribution, imposing a variation on input channel intensities based on their randomization according to the measurement uncertainty estimates at each run. This 
Figure 8 (a) The percentage median absolute relative deviation of the estimated DEM from the model DEM (crosses) and the relative measurement residuals (triangles) as a function of convergence threshold. These are calculated for a 1000 DEMs, with the input measurements varied randomly according to the measurement uncertainty estimates, giving the error bars. (b) The rate of DEM calculations as a function of convergence threshold on a standard desktop PC (see text). (a)
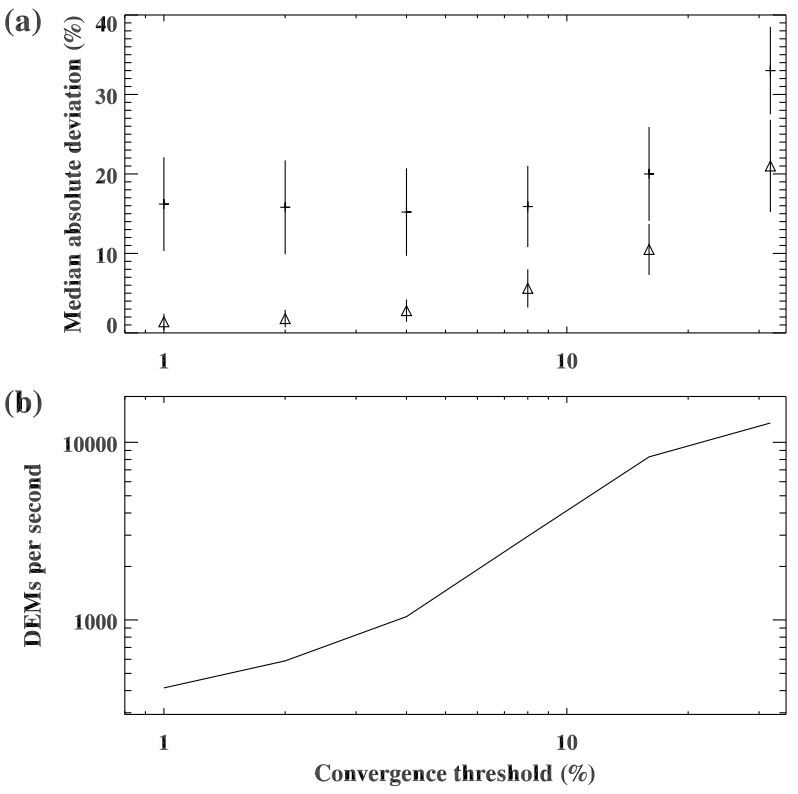

experiment is repeated for convergence thresholds of $1,2,4,8,16$, and $32 \%$. Figure 8a summarizes the performance of SITES as a function of the increasing convergence thresholds through the median absolute residuals of the measurements (goodness of fit) and the median absolute deviation of the resulting DEMs compared to the target model DEM. There is no significant deterioration of achieving the target DEM up to the $8 \%$ convergence threshold. The measurement residuals similarly remain small up to the $8 \%$ convergence threshold. On a Linux desktop Intel Core i7-4790 CPU with 16 Gb memory the 1000 runs are timed with the DEM processing rate shown in Figure $8 \mathrm{~b}$. Based on these results, for real data we set a convergence threshold of $4 \%$, which can process around 1000 DEMs a second. This speed is similar to regularized matrix inversion-based methods such as Hannah and Kontar (2012) or Plowman, Kankelborg, and Martens (2013).

\subsection{Robustness to Noise}

This section tests the reliability of SITES in the presence of noise. A complex DEM formed from three Gaussian peaks and a constant background is used to create synthetic measurements. This DEM is shown as the solid black line in Figure 9a. For a thousand repetitions, the measurements are varied randomly according to a noise amplitude given by the measurement and calibration errors, and the resulting DEMs recorded. The convergence factor is set at $4 \%$, at a value that will typically be used for practical use on real data.

Figure 9a shows the mean DEM, calculated over the thousand repetitions, as a dotted line. This can be compared to the input model DEM, which is shown as a bold solid line. The gray shaded region shows the standard deviation of DEMs over the thousand repetitions. The error bars show the mean DEM errors as calculated by Equation 6. Figure 9b shows the input measurements in each channel, in the absence of noise, as triangle points with the error bars showing the noise level. The cross symbols and error bars show the mean and standard deviation of the fitted measurements (i.e. gained from the output DEM through 
Figure 9 (a) Applying SITES a 1000 times to noise-varying measurements gives a mean DEM (dotted line) and the standard deviation DEM (shaded area) at each temperature bin. The vertical error bars show the estimated error bars gained from Equation 6, averaged over the 1000 experiments. The solid black line is the input model DEM (as described in Section 3.2). (b) The triangle symbols show the input measurements in the absence of noise, with the associated error bars showing the noise amplitude in each channel. The cross symbols and associated error bars show the mean and standard deviation fit to the data over the 1000 cases (gained from the DEMs using Equation 5). (a)

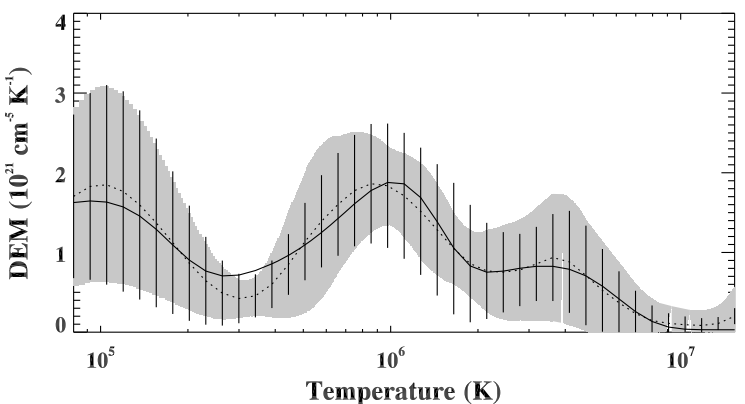

(b)

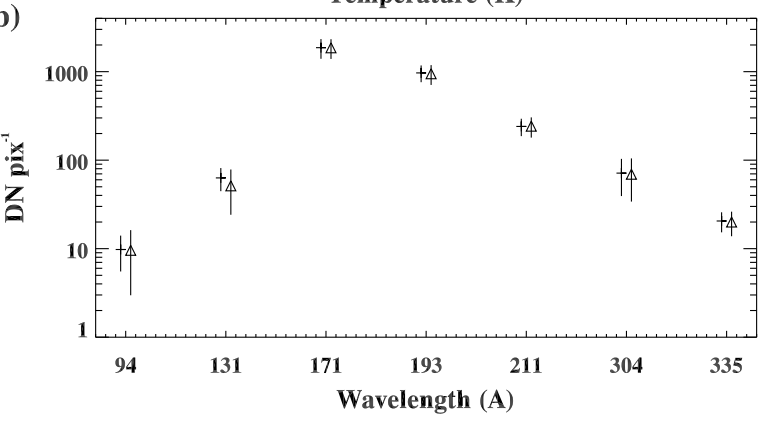

Equation 5). Despite the large variations in the DEM values, the 3-peak profile is well replicated. The presence of noise does not lead to DEMs that deviate significantly beyond that expected given the uncertainties. The uncertainty estimate of Equation 6 reflects well the true variation of the output DEMs. Integrating the product of the DEMs with the response functions (Equation 5) shows that the method is fitting the input data correctly. As can be seen in Figure 9b, the only systematic discrepancy is seen for the low-signal $131 \AA$ A channel, where the method gives a small positive residual.

Figure 10a shows the distribution of DEMs resulting from running the experiment for a signal ten times lower than the previous example. In this very noisy case, SITES performs reasonably well, although the third DEM peak at high temperature is overestimated. The estimated error bars have increased correctly given the increase in noise across temperatures up to $\approx 2 \mathrm{MK}$. Above this temperature, the uncertainty is underestimated. From Figure 10b, the measurement residuals are systematically too high for the lower-signal 94, 131, and $335 \AA$ channels.

\subsection{Comparison with Other Methods}

SITES is compared here with the method of Cheung et al. (2015), hereafter called Sparse Matrix Inversion (SMI), and with the method of Hannah and Kontar (2012), hereafter called Tikhonov Regularization (TR). Both the simple single Gaussian DEM of Section 3.1 and the multiple Gaussian plus constant background DEM of Section 3.2 are used to create synthetic measurements that are given as input to SITES, SMI, and TR. All three methods use identical temperature response functions, measurements, and measurement errors for the inversion. The TR method is called with the default order equal to zero, and we show the positive-constrained solution.

The resulting emissions as functions of temperature for the single Gaussian case are shown in Figure 11a. The result for the default choice of the SMI Gaussian basis functions 
Figure 10 Same as Figure 9, but for the very noisy case of a signal ten times less intense.
Figure 11 Comparison of the input target emission (black line), SITES (red line with error bars), Cheung et al. (2015) (SMI, green lines) and Hannah and Kontar (2012) (blue line with error bars) for (a) the simple single Gaussian DEM of Section 3.1 and (b) the multiple Gaussian DEM of Section 3.2. The SMI method is run for two different values of the width of the Gaussian basis functions (see text). Note that these plots show values of EM rather then DEM, corresponding to the output of the SMI software. (a)

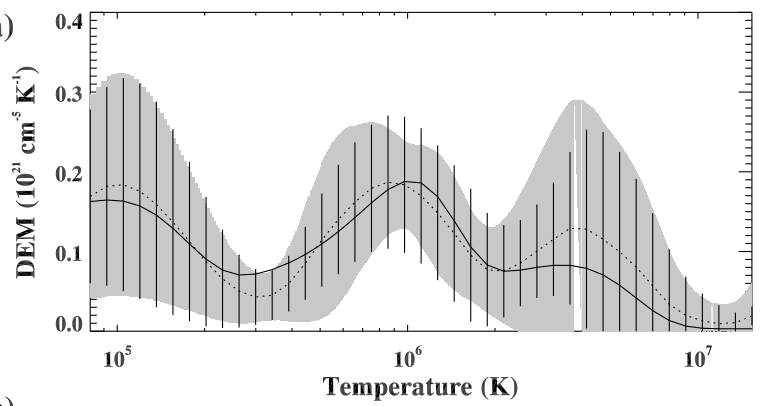

(b)

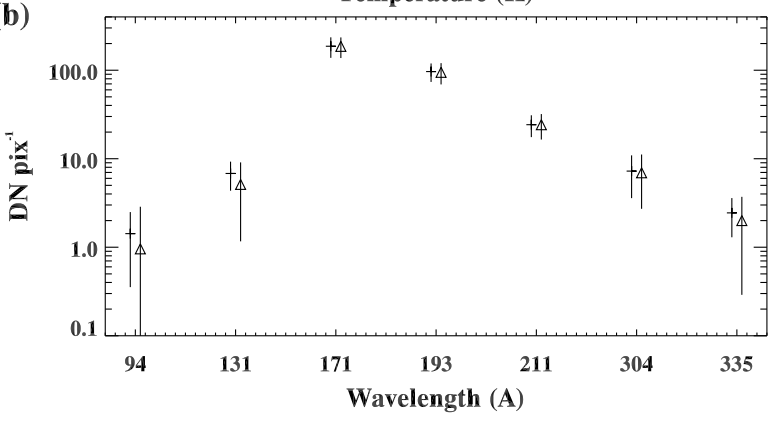

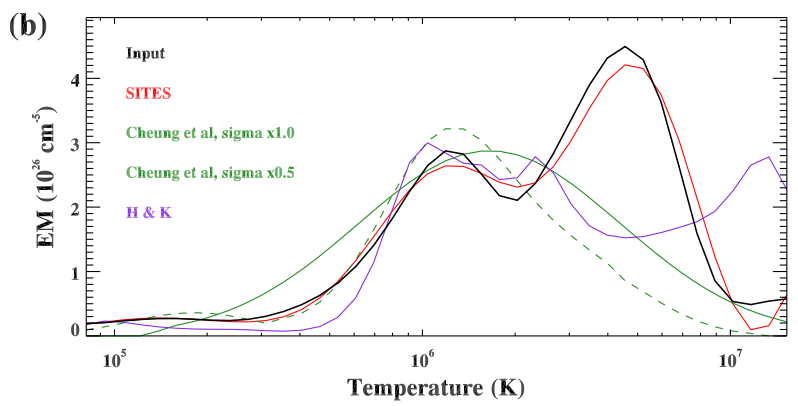

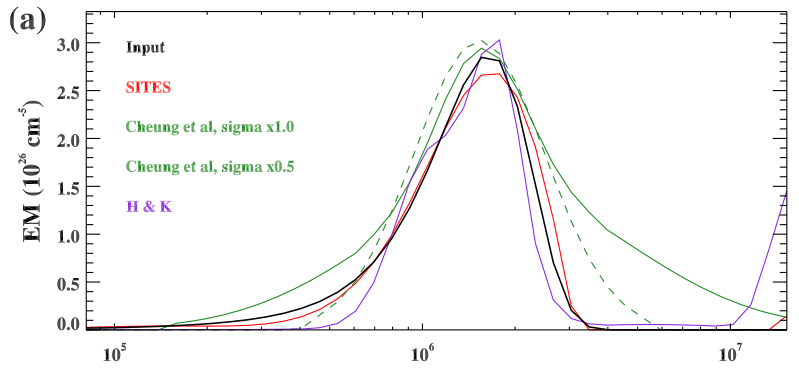

is shown as a solid green line. It is obvious that this choice of basis functions gives an EM result which is too wide. Halving the width of the basis functions (dashed green line) gives a decent fit to the input EM curve, although the emission is too high towards the high-temperature wing of the distribution. TR gives a good fit except at the highest range of temperature, where a steep increase is seen. SITES also has a small increase at the highest 


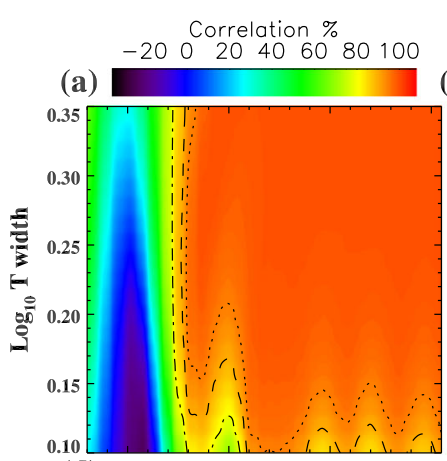

(b)
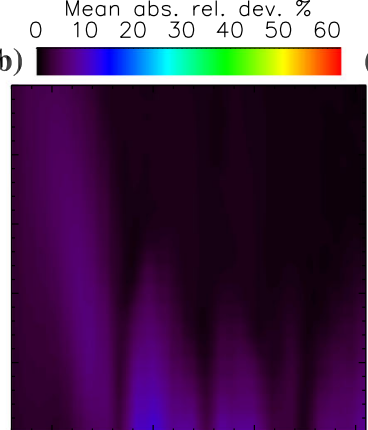

(e)

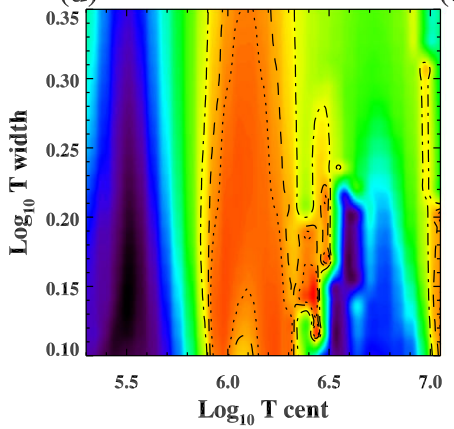

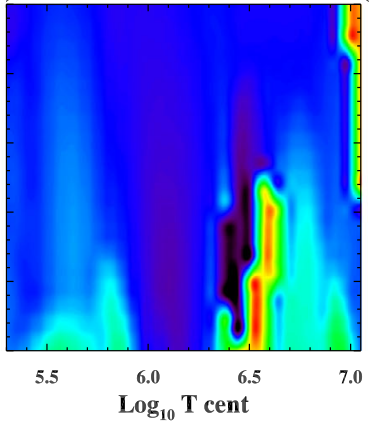

(c)
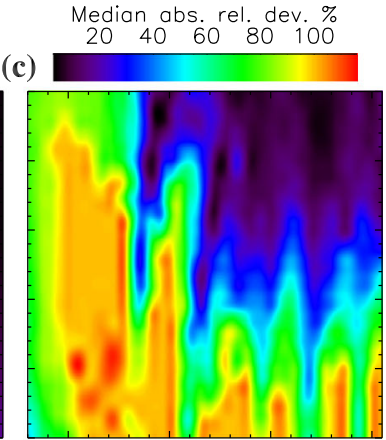

(f)

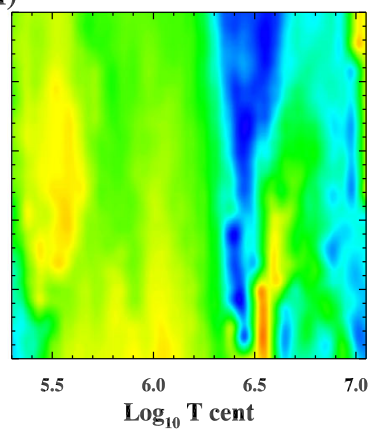

Figure 12 (a) Correlation, $c$, between input and SITES-inverted DEM profiles. (b) Mean absolute relative deviation, $T_{I}$, between input measurement and SITES output fitted measurement. (c) Median absolute relative deviation, $T_{D}$, of input and SITES-inverted DEM profiles. (d) - (f) Same as (a) - (c), but for the TR method. These are calculated for a range of centers and widths in logarithmic temperature of single-Gaussian DEM profiles. The dotted, dashed, and dot-dashed lines in (a) and (d) show the 95, 90 and 80\% correlation levels, respectively. The color bars at the top of each column are common to the plots of both methods.

temperature bin. SITES outperforms both SMI and TR for this example in closely fitting the Gaussian peak and giving zero DEM at higher temperatures.

Figure 11b shows the result for a double-Gaussian input DEM. In the case of using the broad (default) SMI basis functions (solid green line), the estimated EM broadly covers the correct temperature region, but fails to identify the individual peaks. The narrow basis functions (dashed green line) successfully identifies the EM peak near $T=1 \mathrm{MK}$, but fails to invert the other peak, and gives an overall profile which is too narrow across temperature. TR is effective in finding the cooler $T=1 \mathrm{MK}$ peak but fails to identify the main peak near 4 MK. SITES outperforms both SMI and TR for the two-Gaussian DEM profiles in successfully finding all three Gaussian peaks plus the constant background.

The comparison of SITES to the TR method is extended to a parameter search for the case of a single-Gaussian plus background input DEM profile. The parameter space is the same as in Section 3.1, but with a reduced number of bins (30 bins in Gaussian central temperature and 20 bins in Gaussian width). Results are shown in Figure 12, with the top row showing the SITES performance (almost identical to Figure 3, with a different color scale range), and the bottom row showing the TR method performance. The DEM input-output correlation of Figures 12a and $\mathrm{b}$ show a poor inversion for both methods at low temperatures $(\log T<5.7)$. Above this temperature, SITES outperforms TR for almost all central temperatures and widths. SITES also more closely fits the input data by a considerable margin, as shown in Figures $12 \mathrm{~b}$ and e. Figures $12 \mathrm{c}$ and e show the median absolute relative deviation 


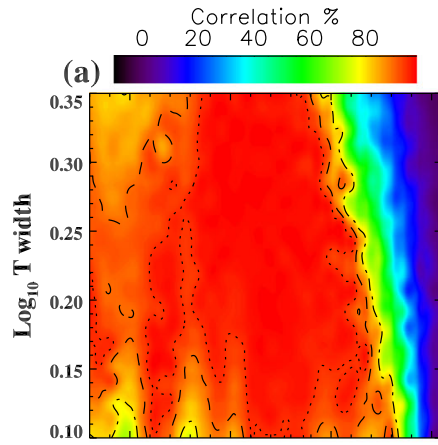

(d)

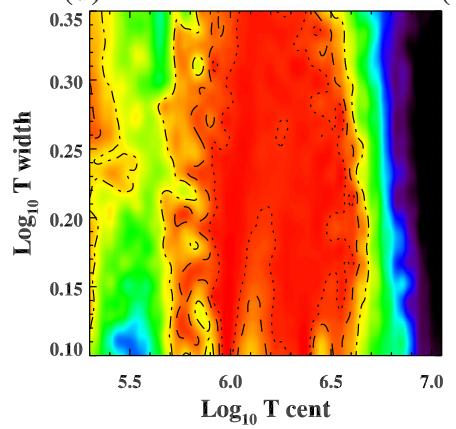

Mean abs. rel. dev. \% $020 \quad 40 \quad 60 \quad 80 \quad 100120$ (b)

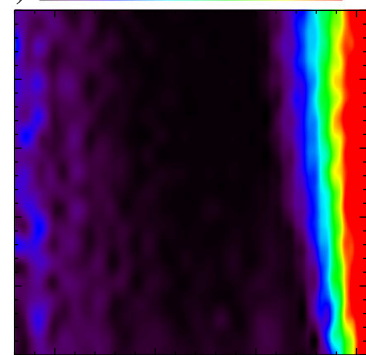

(e)

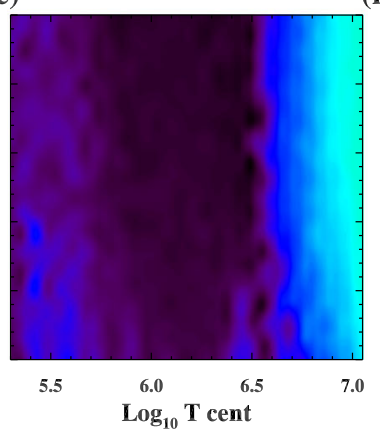

Median abs, rel. dev. \% $40 \quad 60$ 80 100

(c)

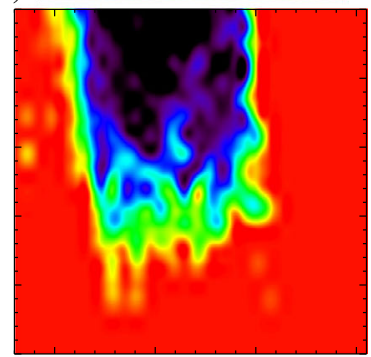

(f)

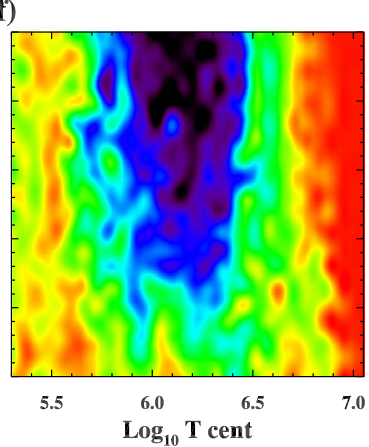

Figure 13 Same as Figure 12, but for the case of input data modulated by Poisson noise. These values show the mean calculated over 15 repetitions with the intensity values varying randomly with an amplitude set by the Poisson uncertainty.

of input and output DEMs for both methods. SITES has a larger region of small deviation $(<40 \%)$ and more profiles that have a very small deviation $(<20 \%)$, but also it has some regions of higher deviation than TR. In summary, SITES generally gives better performance than TR in this noiseless comparison.

As suggested by Figure 12e, the TR method may be underfitting the data, therefore the comparison with SITES may be unfair since the input data has no randomness associated with noise. This is addressed by repeating the test 15 times, allowing the input data to vary randomly according to a Poisson distribution, comparing the output DEM at each repetition to the input DEM and taking the mean correlation, measurement deviation, and DEM deviations over the 15 cases. To give an idea of the noise amplitude, at a central DEM temperature of $\log T=6.4$ and $\log T$ width 0.26 , the relative Poisson noise is $19 \%$ for the lowest signal $94 \AA$ channel and $1.4 \%$ for the $193 \AA$ channel. Results are shown in Figure 13. The input/output DEM correlation is generally better for SITES compared to TR (Figures 13a and d), whilst the DEM deviation (Figures 13c and f) is worse for SITES. In summary, both methods perform similarly for noisy data, with SITES giving an overall better match to the general DEM profiles (a broader region of higher correlation) and TR giving closer absolute values of DEM (a broader region of lower deviation).

We note that we have not investigated with any rigor the various parameters of SMI. We have, for example, only used two choices of the basis function widths. We further note that SMI is extremely fast compared to SITES, around a factor of 100 faster depending on the choice of SITES convergence factor. For the TR method, we have experimented with changing the choice of order (which sets the regularization constraints) with similar results 
Table 1 Some characteristics of a set of AIA observations. The columns show channel, exposure time, $x$-shift (fine alignment relative to the $193 \AA$ channel), $y$-shift, and mean intensity (on the disk).

\begin{tabular}{llrrr}
\hline Channel $(\AA)$ & $t(s)$ & \multicolumn{1}{l}{$x_{s}$} & \multicolumn{1}{l}{$y_{s}$} & $\bar{I}\left(\mathrm{DN} \mathrm{pix}^{-1}\right)$ \\
\hline 94 & 2.9 & 0.86 & -1.47 & 3 \\
131 & 2.9 & 1.59 & -1.10 & 13 \\
171 & 2.0 & -0.37 & -0.62 & 271 \\
193 & 2.0 & - & - & 411 \\
211 & 2.9 & -0.10 & 0.36 & 207 \\
304 & 2.9 & 0.91 & -0.91 & 28 \\
335 & 2.9 & 0.81 & -0.64 & 5 \\
\hline
\end{tabular}

to those shown for order equal to zero. At a convergence threshold of $4 \%$, SITES is of comparable speed to TR.

\section{Application to AIA Data}

\subsection{Data Processing and Error Estimates}

The standard SDO procedure read_sdo.pro is used to open a set of full-resolution images in the seven EUV channels of AIA. An example from 01 January 2015 at 03:00 UT is used here. Figure 14 shows a color composite processed using multiscale Gaussian normalization to provide context (Morgan and Druckmüller, 2014). Each channel image is shifted in the $x$ and $y$ dimensions so that the central pixel corresponds to the solar disk center, as given by the header image geometry information. A secondary sub-pixel fine alignment is achieved through aligning each image to the $193 \AA$ channel image, using a phase correlation method to estimate the required shift (Druckmüller, 2009; Fisher and Welsch, 2008), and cubic interpolation to apply the shift. For the example set of images, these pixel shifts are listed in Table 1. The mean signal calculated over all pixels on the solar disk is listed for each channel in the table.

The uncertainty of the measurements given by the AIA SolarSoftware routine aia_bp_ estimate_error includes the Poisson photon count, dark subtraction, read noise, count quantization, and image compression uncertainties. Figures $15 \mathrm{~b}$ and $\mathrm{c}$ show the range of intensities enclosed by the estimated errors for the $193 \AA$ and $94 \AA$ channels, respectively, for a horizontal cut across the images shown by the dashed red line in Figure 15a. In high-signal regions and channels, the measurement error is small and the dominant uncertainty is in the response functions (calibration uncertainty). In low-signal regions or channels the method is influenced by both the response function and measurement uncertainties. At the expense of spatial and temporal resolution, rebinning images to smaller size through neighborhood averaging and combining two or more consecutive observations over time, will decrease measurement noise in the low signal channels to a more acceptable level.

The dataset of 01 January 2015 is rebinned to $512 \times 512$ pixels, from the original $4096 \times$ 4096 pixels. Since 64 original measurements are combined (averaged) for each pixel, the measurement noise decreases by a factor of $1 / 8$. The DEM method is applied to all pixels at heights below $1.15 R_{\odot}$ and DEMs converted to EM by product with the width of the temperature bins. Emission is shown for four example temperatures in Figure 16.

Effective visualization of DEMs is challenging, since the output result from an imaging instrument is a datacube, thus one can show emission at a given temperature yet the context of emission at other temperatures is absent. Such direct DEM images are also dominated 


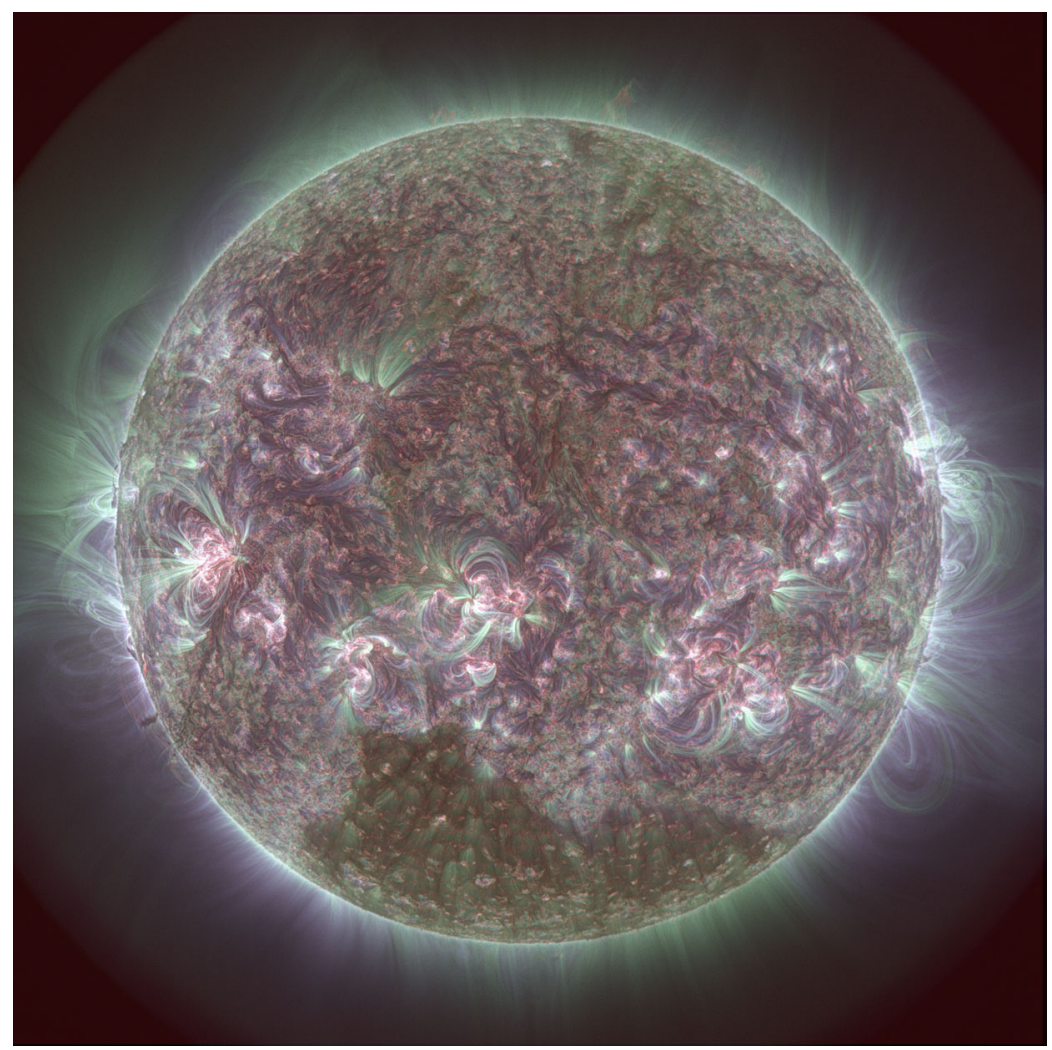

Figure 14 A context image from 01 January 2015 at 03:00 UT. All seven AIA channels contribute to this composite, with the temperature response of each channel between 0.05 and $7.0 \mathrm{MK}$ specifying that channel contribution to the red, green, and blue color channels of the output images. The image is processed with Multiscale Gaussian Normalization to enhance fine-scale structure (Morgan and Druckmüller, 2014).

by the high emission, at all temperatures, of active regions. One effective method is the emission-weighted-mean or median temperature displayed with a color and hue table that can show temperatures and emissions, as shown, for example, in Figure 15 of Plowman, Kankelborg, and Martens (2013). For visually comparing DEM maps in the context of dominance of different regions by certain temperature ranges, we introduce the simple concept of fractional emission measure (FEM). FEM in a temperature bin (indexed $j$ ) is calculated from a DEM by

$$
F E M_{j}=\frac{D E M_{j} \Delta T}{\sum_{j} D E M_{j} \Delta T},
$$

so the FEM in a given temperature bin gives the fraction of emission at that temperature compared to the total emission integrated over all temperatures. FEM maps are shown in Figure 17. These maps, for regions on the disk, are a powerful visualization of the different general temperature dependencies of large-scale coronal features:

i) At $T=0.5 \mathrm{MK}$, the FEM maps are dominated strongly by coronal holes and filament channels. This is an effective way of identifying these regions. 


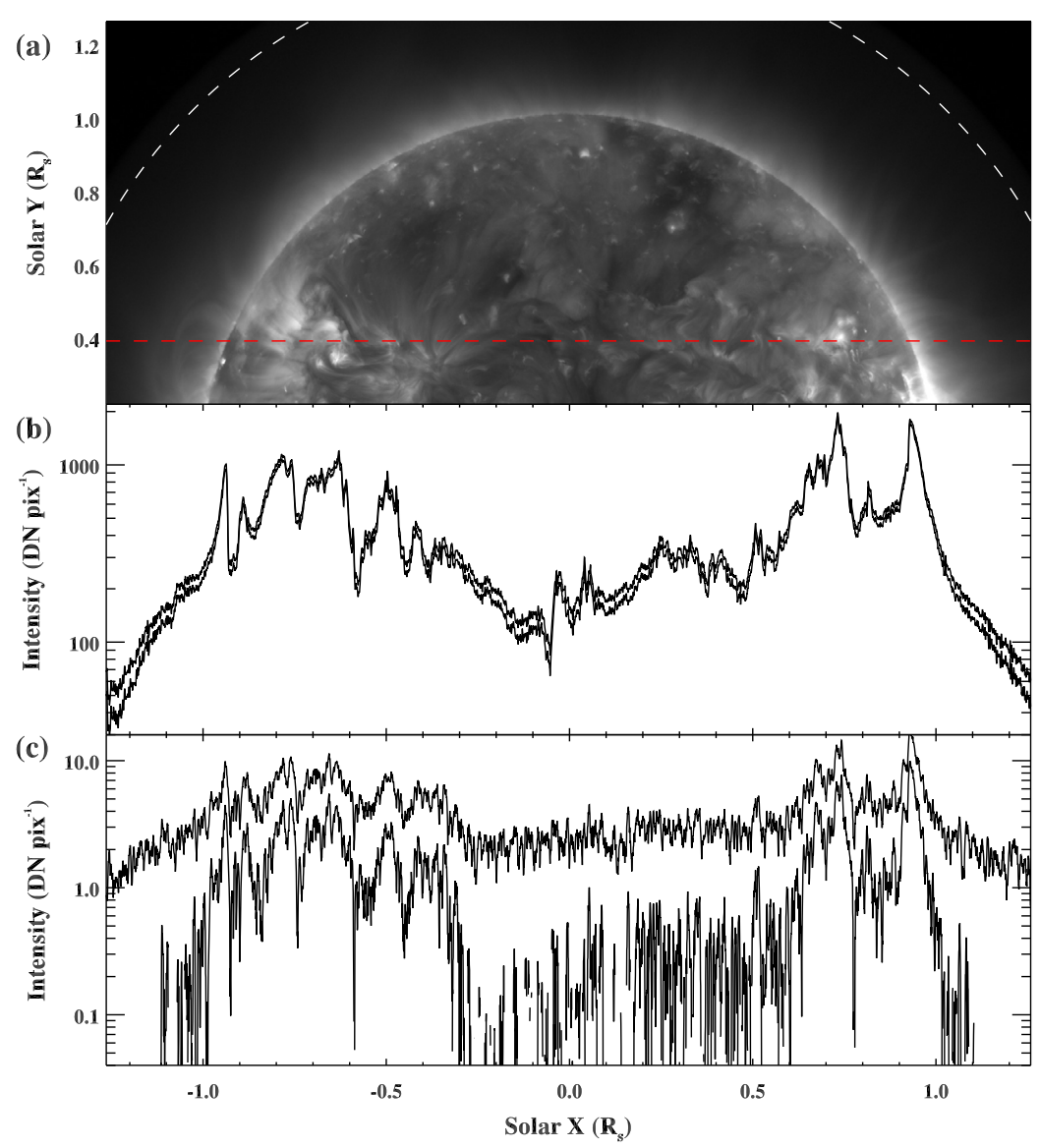

Figure 15 (a) An AIA $193 \AA$ channel image from 01 January 2015 at 03:00 UT. The dashed white line shows a heliocentric height of $1.45 R_{\odot}$ and the dashed red line shows a horizontal cut across the image. (b) The intensity along the dashed red line for the channel with the highest mean intensity (193 $\AA$ ), with the two lines showing the width of the measurement uncertainties. (c) As (b) for the channel with the lowest mean intensity $(94 \AA)$.

ii) At $T=1.5 \mathrm{MK}$, broad regions of the quiet corona and coronal holes have high FEM. Quiet regions surrounding active regions are particularly strong. Note that active regions have generally very low FEM at this temperature.

iii) At $T=4.1 \mathrm{MK}$, all regions except active regions have low FEM. Note in the original EM maps, that active regions have high EM at all temperatures compared to other regions due to their high mass. The FEM maps, through normalization by the total EM, remove this effect and show that, despite the multithermality of active regions, their emission is dominated by high temperatures.

iv) At $T=5.6 \mathrm{MK}$, only the hot cores of the large active regions have high FEM. The quiet coronal regions have close to zero FEM at this temperature.

The DEMs in off-limb regions are hard to interpret and are subject to the bias towards high temperatures with increasing height, given the large height scale for hot structures, as explained by, e.g. Aschwanden (2005). Solar rotational tomography offers a solution to 
(a)

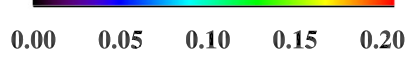

$\mathrm{T}=0.5 \mathrm{MK}$

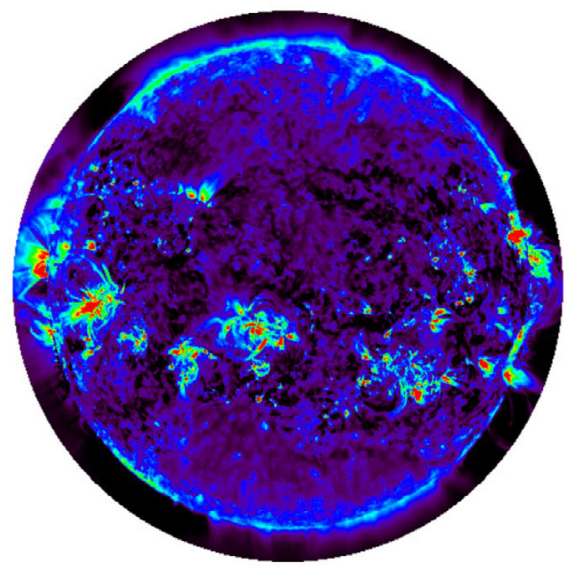

(c)

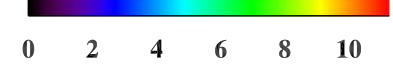

$\mathrm{T}=4.1 \mathrm{MK}$

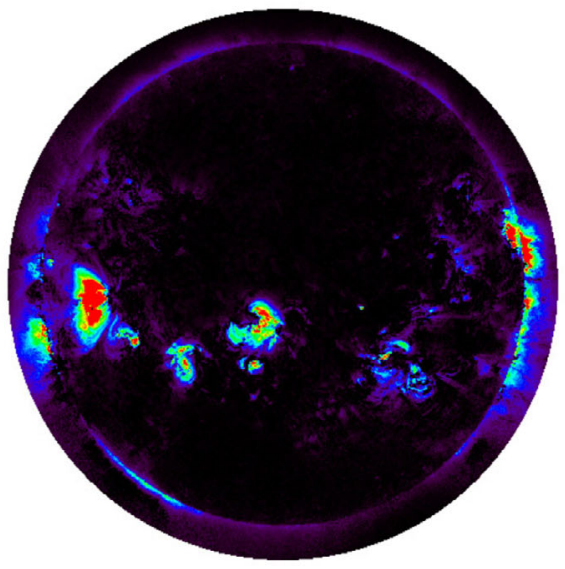

(b)

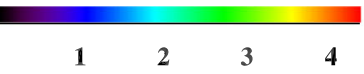

$\mathrm{T}=1.5 \mathrm{MK}$

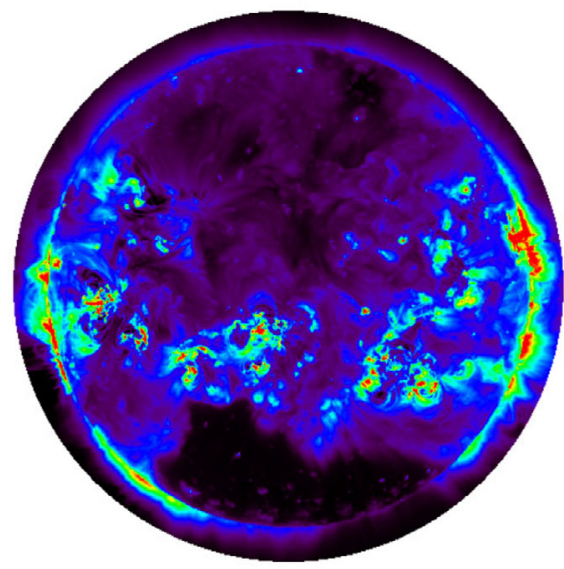

(d)

$\mathrm{T}=5.6 \mathrm{MK}$

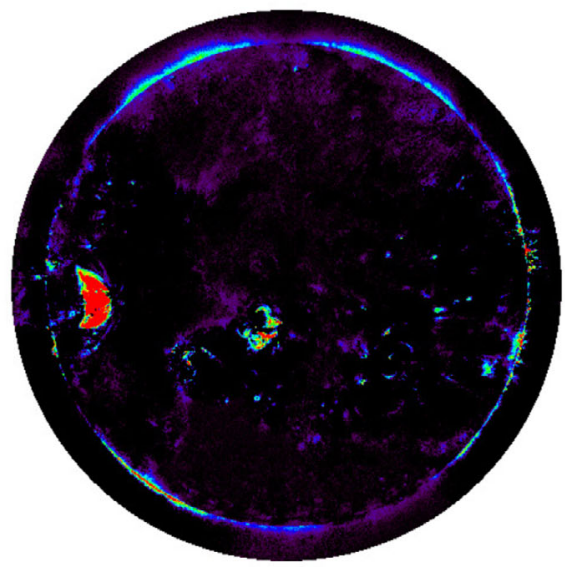

Figure 16 Emission measure (EM) for four different temperatures as indicated in each panel. The field of view is curtailed to a maximum heliocentric distance of $1.15 R_{\odot}$. The color bars give EM in units of $10^{26} \mathrm{~cm}^{-5}$.

this line-of-sight problem. A framework for tomography combined with a DEM analysis is given by Nuevo et al. (2015), where the intensity from each channel, observed from several different viewpoints, is reconstructed in a $3 \mathrm{D}$ volume of emission and a local DEM computed at each voxel.

\section{Summary}

A new DEM method is presented that is reasonably fast, simple in concept, and simple to implement. It performs well on tests involving model DEMs and synthetic data based on 
(a)

$\begin{array}{llllll}0.00 & 0.05 & 0.10 & 0.15 & 0.20 & 0.25\end{array}$

$\mathrm{T}=0.5 \mathrm{MK}$

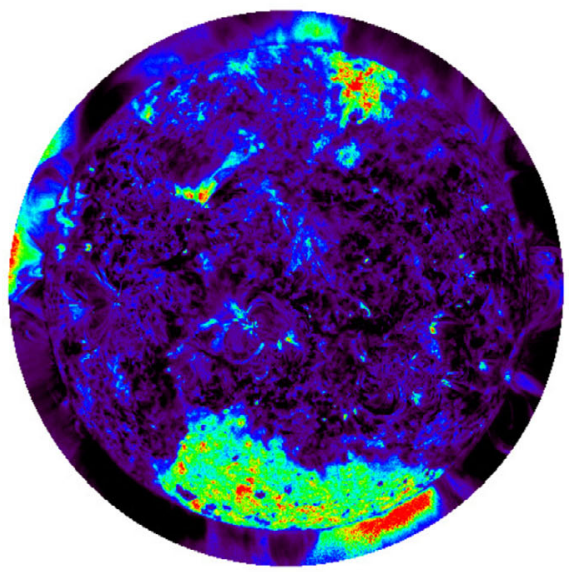

(c)
$\begin{array}{lllllll}0.0 & 0.5 & 1.0 & 1.5 & 2.0 & 2.5 & 3.0\end{array}$

\section{$\mathrm{T}=4.1 \mathrm{MK}$}

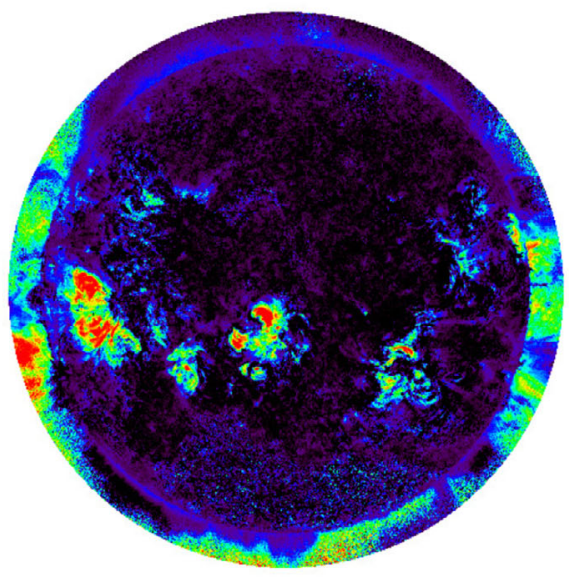

(b)

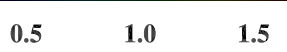

$\mathrm{T}=1.5 \mathrm{MK}$

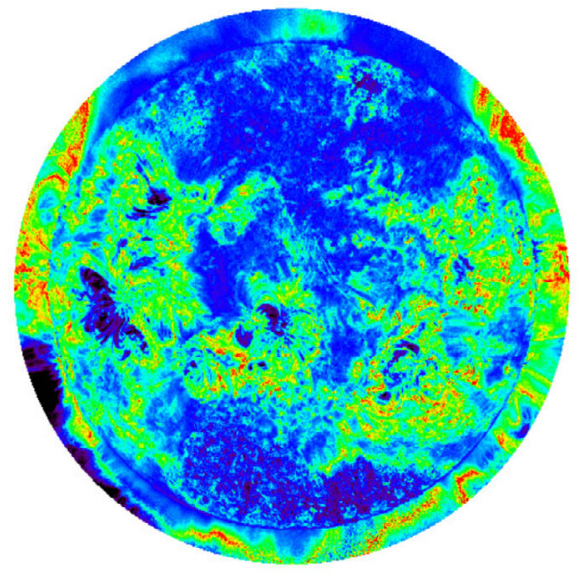

(d)

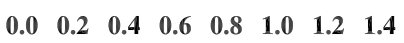

$\mathrm{T}=5.6 \mathrm{MK}$

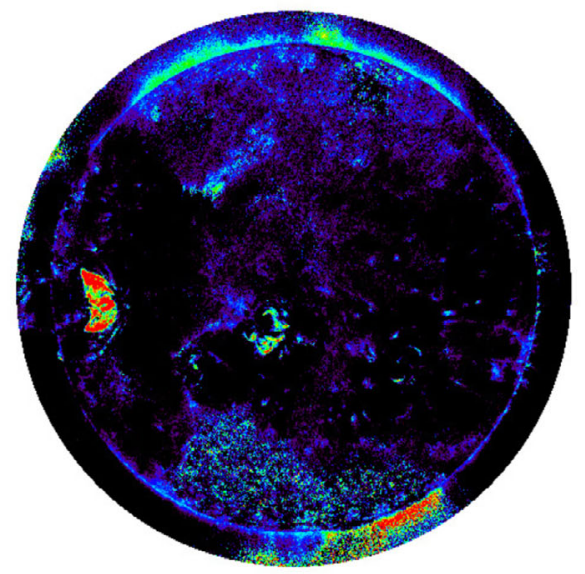

Figure 17 Fractional emission (FEM) for four different temperatures as indicated in each panel. The field of view is curtailed to a maximum heliocentric distance of $1.15 R_{\odot}$. The color bars give FEM in $\%$.

the AIA/SDO instrument. In particular, the correlation between the model input DEMs and SITES inversions is excellent for a broad range of coronal temperatures. SITES performs less well on very narrow DEM peaks, and performs very poorly for temperatures below $\approx 0.5 \mathrm{MK}$. This weakness is likely due to the limitations of the AIA/SDO instrumental temperature response curves rather than the SITES inversion itself, since other inversion methods show the same failing.

Applied to a set of AIA/SDO observations of the full-disk corona, SITES gives sensible values of emission measure as a function of temperature. Fractional emission measure is introduced as a simple yet powerful method to visualize DEM results within images, enabling straightforward comparison of different temperature regimes between regions. 
The computational speed of the method compares well with most methods, but cannot compete with the sparse matrix approach of Cheung et al. (2015). However, the main advantages of SITES is its simplicity of concept and application, and its non-subjectiveness. Equations 4 and 5 form the core of the iterative procedure and are simple to implement. The results of any DEM inversion method are subject to choices of the fitting parameters. In the case of SITES, there is only one of the parameters which affects the result - the width of the smoothing kernel. Thus the method is relatively non-subjective.

The incentive for developing the method is to analyze large datasets, thus enabling largescale studies of coronal changes over long time-scales using AIA/SDO. The method has therefore not been tested on flare-like temperatures. Reliable studies of such high temperatures need measurements by other instruments, possibly in combination with AIA/SDO. Given a set of temperature response functions and error estimates, the method presented here should work reliably - this will be investigated in the near future.

Future work by the authors (paper in preparation) involves a gridding method that may be used with any DEM inversion method to increase computational efficiency by one or two orders of magnitude. This will enable rapid processing of large datasets for AIA/SDO and other current or future instruments. The software for the DEM fitting method of this paper, plus the FEM visualization method, written in IDL, is available by email request to the authors.

Acknowledgements James Pickering is supported by an STFC studentship. Part of Huw Morgan's work on this project is supported by an STFC consolidated grant to Aberystwyth University. CHIANTI is a collaborative project involving George Mason University, the University of Michigan (USA), University of Cambridge (UK), and NASA Goddard Space Flight Center (USA).

Disclosure of Potential Conflict of Interest The authors declare that they have no conflict of interest.

Publisher's Note Springer Nature remains neutral with regard to jurisdictional claims in published maps and institutional affiliations.

Open Access This article is distributed under the terms of the Creative Commons Attribution 4.0 International License (http://creativecommons.org/licenses/by/4.0/), which permits unrestricted use, distribution, and reproduction in any medium, provided you give appropriate credit to the original author(s) and the source, provide a link to the Creative Commons license, and indicate if changes were made.

\section{References}

Aschwanden, M.J.: 2005, Physics of the Solar Corona. An Introduction with Problems and Solutions, 2nd edn. Springer, Berlin.

Boerner, P.F., Testa, P., Warren, H., Weber, M.A., Schrijver, C.J.: 2014, Photometric and thermal crosscalibration of solar EUV instruments. Solar Phys. 289(6), 2377. DOI.

Cheung, M.C.M., Boerner, P., Schrijver, C.J., Testa, P., Chen, F., Peter, H., Malanushenko, A.: 2015, Thermal Diagnostics with the Atmospheric Imaging Assembly on board the Solar Dynamics Observatory: A Validated Method for Differential Emission Measure Inversions. Astrophys. J. 807, 143. DOI.

Del Zanna, G.: 2013, The multi-thermal emission in solar active regions. Astron. Astrophys. 558, A73. DOI.

Dere, K.P., Landi, E., Mason, H.E., Monsignori Fossi, B.C., Young, P.R.: 1997, CHIANTI - An atomic database for emission lines. Astron. Astrophys. Suppl. 125, 149. DOI.

Druckmüller, M.: 2009, Phase Correlation Method for the Alignment of Total Solar Eclipse Images. Astrophys. J. 706, 1605. DOI.

Fisher, G.H., Welsch, B.T.: 2008, FLCT: A Fast, Efficient Method for Performing Local Correlation Tracking. In: Howe, R., Komm, R.W., Balasubramaniam, K.S., Petrie, G.J.D. (eds.) Subsurface and Atmospheric Influences on Solar Activity, Astron. Soc. Pacific Conf. Ser. 383, 373.

Hahn, M., Landi, E., Savin, D.W.: 2011, Differential Emission Measure Analysis of a Polar Coronal Hole during the Solar Minimum in 2007. Astrophys. J. 736, 101. DOI. 
Hahn, M., Savin, D.W.: 2014, Evidence for Wave Heating of the Quiet-Sun Corona. Astrophys. J. 795, 111. DOI.

Hannah, I.G., Kontar, E.P.: 2012, Differential emission measures from the regularized inversion of Hinode and SDO data. Astron. Astrophys. 539, A146. DOI.

Landi, E., Del Zanna, G., Young, P.R., Dere, K.P., Mason, H.E.: 2012, CHIANTI - An Atomic Database for Emission Lines. XII. Version 7 of the Database. Astrophys. J. 744, 99. DOI.

Mackovjak, Š., Dzifčáková, E., Dudík, J.: 2014, Differential emission measure analysis of active region cores and quiet Sun for the non-Maxwellian $\kappa$-distributions. Astron. Astrophys. 564, A130. DOI.

Morgan, H., Druckmüller, M.: 2014, Multi-scale Gaussian normalization for solar image processing. Solar Phys. 289(8), 2945. DOI.

Morgan, H., Taroyan, Y.: 2017, Global conditions in the solar corona from 2010 to 2017. Sci. Adv. 3(7), e1602056. DOI. https://advances.sciencemag.org/content/3/7/e1602056.

Nuevo, F.A., Vásquez, A.M., Landi, E., Frazin, R.: 2015, Multimodel differential emission measure in the solar corona. Astrophys. J. 811(2), 128. DOI.

Plowman, J., Kankelborg, C., Martens, P.: 2013, Fast Differential Emission Measure Inversion of Solar Coronal Data. Astrophys. J. 771, 2. DOI.

Weber, M.A., Schmelz, J.T., DeLuca, E.E., Roames, J.K.: 2005, Isothermal Bias of the "Filter Ratio" Method for Observations of Multithermal Plasma. Astrophys. J. Lett. 635, 101. DOI. 